\title{
Transmission networks of HIV-1 among men who have sex with men in east and southeast Asia
}

\author{
Kok Keng Tee, $\mathrm{PhD}^{1}$, Rami Kantor, $\mathrm{MD}^{2}$, Somnuek Sungkanuparph, $\mathrm{MD}^{3}$, Yutaka Takebe, \\ MD, PhD ${ }^{1,4}$, Patrick Li, MBBS ${ }^{5}$, Rossana Ditangco, MD ${ }^{6}$, Praphan Phanuphak, MD, PhD ${ }^{7}$, \\ Thira Sirisanthana, $\mathbf{M D}^{8}$, Benedict Sim, MD, PhD $^{9}$, Winai Ratanasuwan, MD ${ }^{10}$, Pacharee \\ Kantipong, MD $^{11}$, and Adeeba Kamarulzaman, MBBS, FRACP ${ }^{1}$ on behalf of the TREAT Asia \\ Studies to Evaluate Resistance - Monitoring (TASER-M) Database
}

\begin{abstract}
${ }^{1}$ Centre of Excellence for Research in AIDS (CERiA), Department of Medicine, Faculty of Medicine, University of Malaya, Kuala Lumpur, Malaysia ²Division of Infectious Diseases, Department of Medicine, Alpert Medical School of Brown University, Providence, RI, USA ${ }^{3}$ Department of Medicine, Faculty of Medicine, Ramathibodi Hospital, Mahidol University, Bangkok, Thailand ${ }^{4}$ AIDS Research Center, National Institute of Infectious Diseases, Toyama, Shinjuku-ku, Tokyo, Japan ${ }^{5}$ Queen Elizabeth Hospital, Hong Kong, China ${ }^{6}$ Research Institute for Tropical Medicine, Manila, Philippines ${ }^{7}$ HIV-NAT/Thai Red Cross AIDS Research Centre, Bangkok, Thailand ${ }^{8}$ Research Institute for Health Sciences, Chiang Mai University, Chiang Mai, Thailand ${ }^{9} \mathrm{Hospital}$ Sungai Buloh, Kuala Lumpur, Malaysia ${ }^{10}$ Faculty of Medicine Siriraj Hospital, Mahidol University, Bangkok, Thailand ${ }^{11}$ Chiangrai Prachanukroh Hospital, Chiang Rai, Thailand
\end{abstract}

\section{To the Editors}

The HIV epidemic among men who have sex with men (MSM) in Asia is expanding at an alarming rate especially in several urban centres. ${ }^{1,2}$ Understanding the dynamics of HIV-1 transmission among MSM across large geographic areas may provide essential information on the origin of viral lineages and their epidemic expansion. Such estimates provide insights on the size and frequency of the disease spread, which can be used to facilitate the implementation of intervention strategies for prevention, such as the use of pre-exposure prophylaxis (PrEP) and combination antiretroviral therapy (cART). ${ }^{3,4}$ The availability of large datasets of genetic sequences from antiretroviral resistance surveillance activities and the use of phylodynamic tools allow for the identification and characterization of transmission clusters. ${ }^{5,6}$

In this study, we characterized the transmission clusters of HIV-1 among MSM across countries in East and Southeast Asia. A total of 1,856 HIV-1 polymerase gene sequences from HIV-infected individuals were obtained from the TREAT Asia Studies to Evaluate Resistance - Monitoring (TASER-M) Database between 2006 and 2011. The details about this study has been described previously. ${ }^{7}$ Briefly, patients initiating first-line cART from

*Correspondence: Centre of Excellence for Research in AIDS (CERiA), Department of Medicine, Faculty of Medicine, University of Malaya, 50603 Kuala Lumpur, Malaysia. Phone: +60-3-7967-7833; Fax: +60-3-7967-7834; k2tee@um.edu.my.

Potential conflicts of interest: The authors have no conflicts of interest to disclose. 
participating urban hospitals in Hong Kong, Thailand, Malaysia, and the Philippines were included. Ethics approvals were obtained from the local institutional review boards and informed consent was obtained prior to baseline genotypic resistance testing. Polymerase genes were sequenced (HXB2 position: 2,252-3,263 nt) in laboratories participating in the TREAT Asia Quality Assurance Scheme (TAQAS) ${ }^{8}$ using in-house and/or commercially available resistance assays on specimens collected within 6 months prior to initiating cART. A total of 364 antiretroviral-naive MSM sequences were identified from the TASER-M database (Hong Kong, $n=118$; Thailand, $n=124$; Malaysia, $n=56$; Philippines, $n=66$ ). Following manual inspection (to remove problematic sequences) and phylogenetic inference, 144 subtype B and 186 CRF01_AE sequences from antiretroviral-naive MSM were identified and subjected to phylodynamic analysis. Unique recombinant forms and other rare subtypes $(n=29)$ were excluded from further analysis. Global MSM reference sequences of HIV-1 subtype B $(n=362)$ and CRF01_AE $(n=40)$ were retrieved from the HIV sequence database, ${ }^{9}$ in which sequences from other Asian countries (China, Mongolia and Myanmar) were also included. Transmission clusters from the time-stamped sequence datasets were first deduced by neighbour-joining tree reconstruction followed by the more robust maximum likelihood and Bayesian maximum clade credibility (MCC) inference implemented in PAUP version $4.0^{10}$ and BEAST $1.7,{ }^{11}$ respectively. Transmission cluster was defined based on the recently reported criteria: a phylogenetic cluster consisting of at least 2 isolates that form a clade supported by high bootstrap values (>90\%) and Bayesian posterior probability value of 1 at the tree node. ${ }^{12-14}$ The divergence times or time of the most recent common ancestor (tMRCA) for each subtype B and CRF01_AE transmission clusters were estimated using the Bayesian coalescent methods as described previously. ${ }^{15}$

Phylogenetic reconstructions of the TASER-M datasets showed that a total of $68.1 \%$ of HIV-1 subtype B and $45.7 \%$ of CRF01_AE sequences were grouped in 50 transmission clusters of various sizes (mean size $=5.6$, range $=2-32$ sequences), with subtype B sequences having a higher tendency to form a cluster $(p<.0001)$. Together with other Asian sequences from China, Mongolia and Myanmar, a total of 34 clusters involving 154 subtype B-infected individuals and 16 clusters involving 125 subjects infected with CRF01_AE were estimated (Figure 1A). In both subtypes, most clusters contained individuals from the same geographical origin (i.e. country), although about $22.0 \%$ of the clusters comprised of individuals from more than one country. This suggests that MSM networks in East and Southeast Asia were usually localized in their respective countries, with some clusters spanning beyond a single country. Genealogy-based analysis to estimate the tMRCA for each transmission network indicated the continued emergence of new subtype $\mathrm{B}$ and CRF01_AE clusters in the past three decades (Figure 1B). The uninterrupted growth of each sub-epidemics of various cluster sizes suggest the role of transmission clusters as the continuous driving force of the epidemic among MSM in Asia.

Information on HIV-1 transmission clusters generated by identifying genetically close virus variants circulating at a population level provides real-time, direct evidence of an ongoing forward transmission that otherwise cannot be readily detected by conventional epidemiological surveillance. Despite expanded access to cART in some developing and developed nations in Asia, our analysis showed continued emergence of recent HIV-1 
subtype B and CRF01_AE networks compared to the period in the 1980s and early 1990s where cART was less accessible. Although incomplete sampling or cluster extinction (deadend transmission) may influence our interpretation, ${ }^{16}$ and the fact that most of the TASERM datasets were sampled primarily in 2008-2009 (62.4\%), the observed trend suggests that increased access to cART in general may not be sufficient in reducing transmission clusters. The seemingly reduced transmission rates among individuals on cART could have been outpaced by the rapidly growing networks among undiagnosed and untreated MSM. Therefore, strategies such as early diagnosis and initiation of cART to reduce the risk of transmission among sero-discordant partners need to be urgently implemented and expanded across the region. ${ }^{3,4}$ The overall success of prevention or even harm reduction methods can in turn be assessed by monitoring the development of transmission networks, where in the presence of effective transmission control strategy a significant decrease in the size and number of clusters over time should be observed.

\section{Acknowledgments}

We thank the patients for their participations in this study.

Funding: The TREAT Asia Studies to Evaluate Resistance (TASER) is an initiative of TREAT Asia, a program of amfAR, The Foundation for AIDS Research, with major support provided by the Dutch Ministry of Foreign Affairs through a partnership with Stichting Aids Fonds, and with additional support from amfAR and the National Institute of Allergy and Infectious Diseases (NIAID) of the U.S. National Institutes of Health (NIH) and the National Cancer Institute (NCI) as part of the International Epidemiologic Databases to Evaluate AIDS (IeDEA) (grant no. U01AI069907). Queen Elizabeth Hospital and the Integrated Treatment Centre are supported by the Hong Kong Council for AIDS Trust Fund. The Kirby Institute is funded by the Australian Government Department of Health and Ageing, and is affiliated with the Faculty of Medicine, UNSW Australia (The University of New South Wales). The content of this publication is solely the responsibility of the authors and does not necessarily represent the official views of any of the governments or institutions mentioned above. A.K. and K.K.T. received funding from the Ministry of Education, Malaysia (High Impact Research E-000001-20001 to A.K. and H-500001-00-A000012-000001 to K.K.T.)

\section{Members of the TASER study}

PCK Li ${ }^{I * *}$ and MP Lee, Queen Elizabeth Hospital and KH Wong, Integrated Treatment Centre, Hong Kong, China; N Kumarasamy ${ }^{* \S}$ and S Saghayam, YRGCARE Medical Centre, Chennai, India; S Pujari* and K Joshi, Institute of Infectious Diseases, Pune, India; TP Merati* and F Yuliana, Faculty of Medicine, Udayana University \& Sanglah Hospital, Bali, Indonesia; CKC Lee and BLH Sim*, Hospital Sungai Buloh, Kuala Lumpur, Malaysia; A Kamarulzaman ${ }^{*}$ Il and LY Ong, University of Malaya Medical Centre, Kuala Lumpur, Malaysia; M Mustafa ${ }^{*}$ and N Nordin, Hospital Raja Perempuan Zainab II, Kota Bharu, Malaysia; R Ditangco ${ }^{* \dagger}$ and RO Bantique, Research Institute for Tropical Medicine, Manila, Philippines; YMA Chen ${ }^{*}$ and YT Lin, Kaohsiung Medical University, Kaohsiung City, Taiwan; P Phanuphak* and S Sirivichayakul, HIV-NAT/Thai Red Cross AIDS Research Centre, Bangkok, Thailand; S Sungkanuparph", S Kiertiburanakul, and L Chumla, Faculty of Medicine Ramathibodi Hospital, Mahidol University, Bangkok, Thailand; T Sirisanthana ${ }^{*}$ and J Praparattanapan, Research Institute for Health Sciences, Chiang Mai

IProtocol Co-Chair

*TASER Steering Committee member;

\$rotocol Chair;

†Steering Committee Chair; 
University, Chiang Mai, Thailand; P Kantipong* and P Kambua, Chiangrai Prachanukroh Hospital, Chiang Rai, Thailand; W Ratanasuwan* and R Sriondee, Faculty of Medicine Siriraj Hospital, Mahidol University, Bangkok, Thailand; R Kantor*, Brown University, Rhode Island, U.S.A.; AH Sohn, N Durier* and T Singtoroj, TREAT Asia, amfAR - The Foundation for AIDS Research, Bangkok, Thailand; DA Cooper, MG Law* , A Jiamsakul, and DC Boettiger, The Kirby Institute, UNSW Australia, Sydney NSW, Australia.

\section{References}

1. Beyrer C, Baral SD, van Griensven F, et al. Global epidemiology of HIV infection in men who have sex with men. Lancet. 2012; 380:367-377. [PubMed: 22819660]

2. Mayer KH. The next tsunami? HIV spread in Asian men who have sex with men. Clin Infect Dis. 2014; 58:1760-1762. [PubMed: 24647018]

3. Cohen MS, Chen YQ, McCauley M, et al. Prevention of HIV-1 infection with early antiretroviral therapy. N Engl J Med. 2011; 365:493-505. [PubMed: 21767103]

4. Grant RM, Lama JR, Anderson PL, et al. Preexposure chemoprophylaxis for HIV prevention in men who have sex with men. N Engl J Med. 2010; 363:2587-2599. [PubMed: 21091279]

5. Hughes GJ, Fearnhill E, Dunn D, Lycett SJ, Rambaut A, Leigh Brown AJ. Molecular phylodynamics of the heterosexual HIV epidemic in the United Kingdom. PLoS Pathog. 2009; 5:e1000590. [PubMed: 19779560]

6. Lewis F, Hughes GJ, Rambaut A, Pozniak A, Leigh Brown AJ. Episodic sexual transmission of HIV revealed by molecular phylodynamics. PLoS Med. 2008; 5:e50. [PubMed: 18351795]

7. Sungkanuparph S, Oyomopito R, Sirivichayakul S, et al. HIV-1 drug resistance mutations among antiretroviral-naive HIV-1-infected patients in Asia: results from the TREAT Asia Studies to Evaluate Resistance-Monitoring Study. Clin Infect Dis. 2011; 52:1053-1057. [PubMed: 21460324]

8. Land S, Zhou J, Cunningham P, et al. Capacity building and predictors of success for HIV-1 drug resistance testing in the Asia-Pacific region and Africa. J Int AIDS Soc. 2013; 16:18580. [PubMed: 23845227]

9. Los Alamos HIV Sequence Database. http://www.hiv.lanl.gov

10. PAUP* Phylogenetic Analysis Using Parsimony (*and Other Methods). Sinauer Associates; Sunderland, Massachusetts: 2003. [computer program]. Version 4.0 beta

11. Drummond AJ, Rambaut A. BEAST: Bayesian evolutionary analysis by sampling trees. BMC Evol Biol. 2007; 7:214. [PubMed: 17996036]

12. Aldous JL, Pond SK, Poon A, et al. Characterizing HIV transmission networks across the United States. Clin Infect Dis. 2012; 55:1135-1143. [PubMed: 22784872]

13. Chalmet K, Staelens D, Blot S, et al. Epidemiological study of phylogenetic transmission clusters in a local HIV-1 epidemic reveals distinct differences between subtype B and non-B infections. BMC Infect Dis. 2010; 10:262. [PubMed: 20822507]

14. Zehender G, Ebranati E, Lai A, et al. Population dynamics of HIV-1 subtype B in a cohort of menhaving-sex-with-men in Rome, Italy. J Acquir Immune Defic Syndr. 2010; 55:156-160. [PubMed: 20703157]

15. Ng KT, Ong LY, Lim SH, Takebe Y, Kamarulzaman A, Tee KK. Evolutionary history of HIV-1 subtype B and CRF01_AE transmission clusters among men who have sex with men (MSM) in Kuala Lumpur, Malaysia. PLoS One. 2013; 8:e67286. [PubMed: 23840653]

16. Abecasis AB, Lemey P, Vidal N, et al. Recombination confounds the early evolutionary history of human immunodeficiency virus type 1: subtype $\mathrm{G}$ is a circulating recombinant form. J Virol. 2007; 81:8543-8551. [PubMed: 17553886] 
(A)

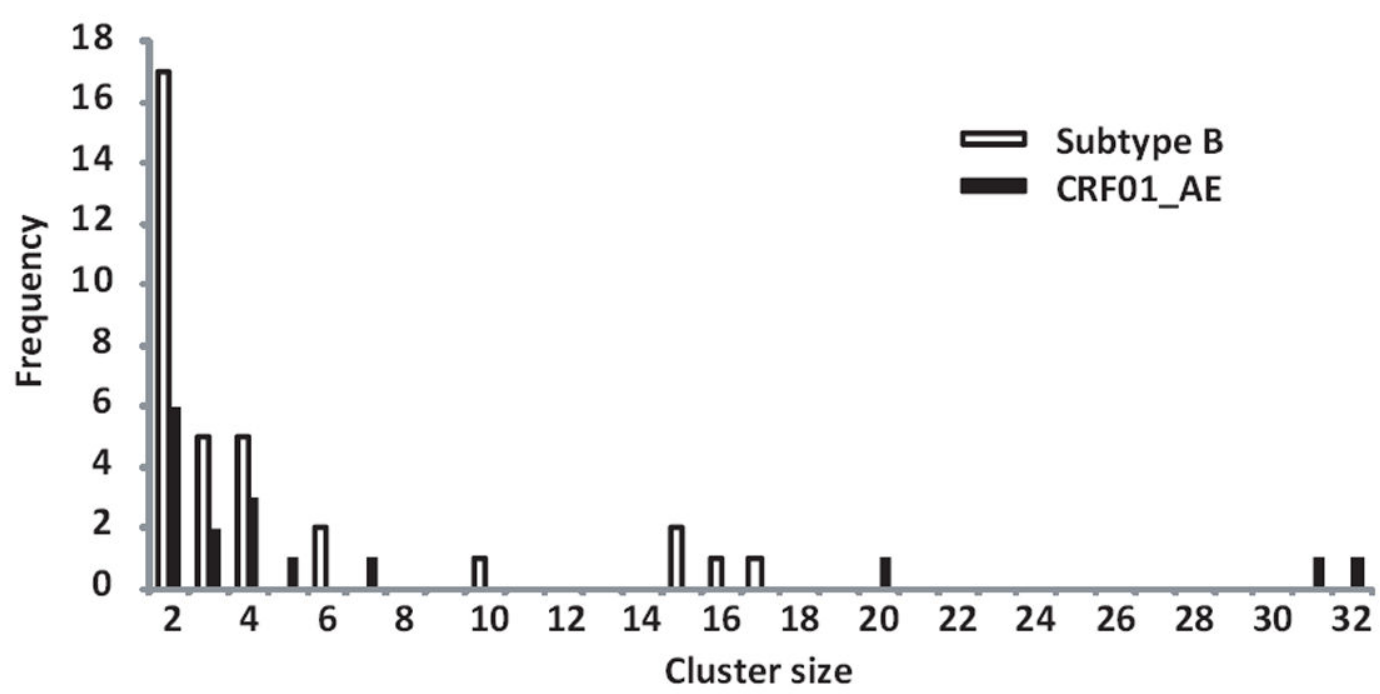

(B)

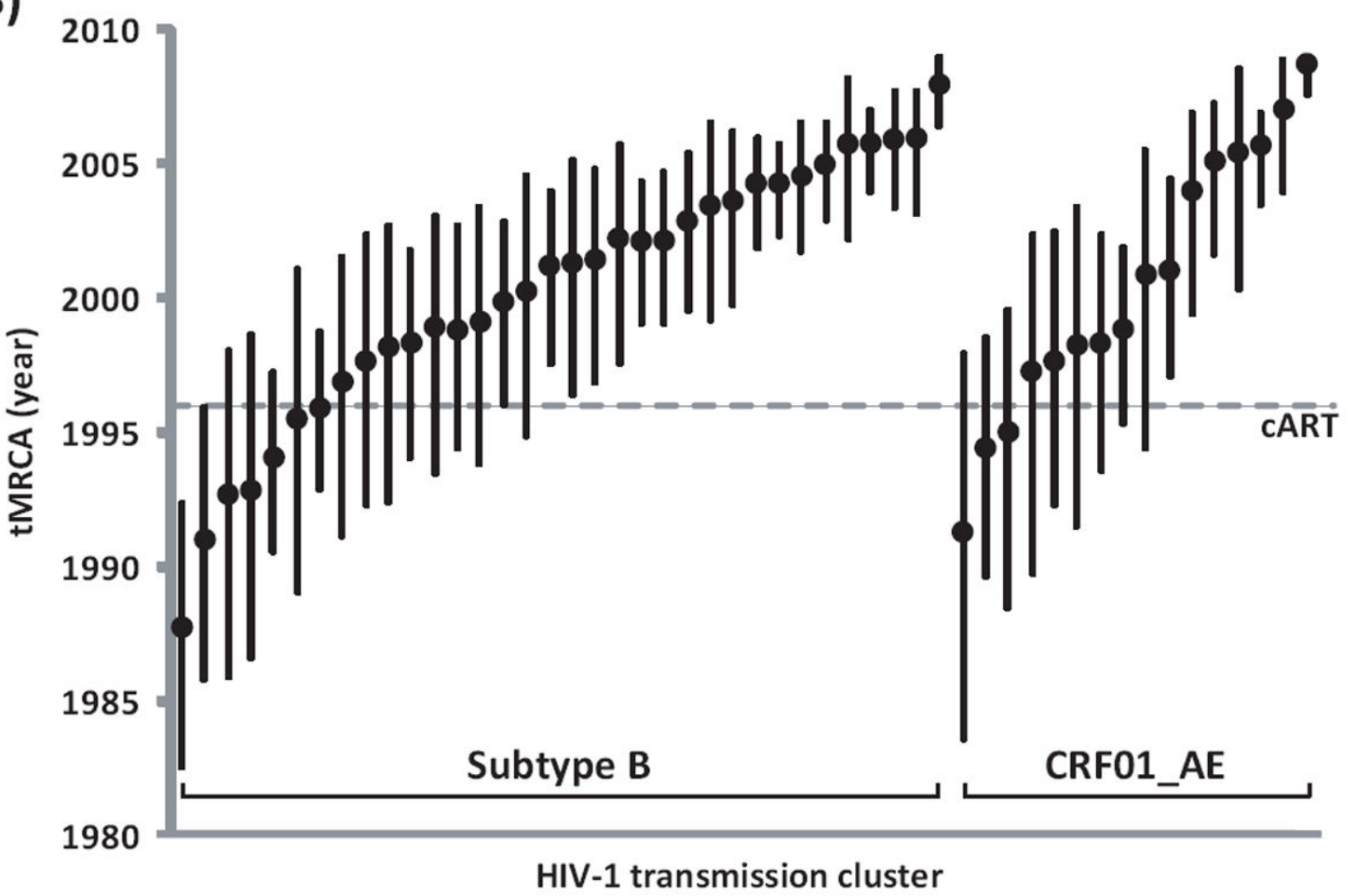

Figure 1. Transmission clusters and phylodynamic profile of HIV-1 subtype B and CRF01_AE among men who have sex with men (MSM) in East and Southeast Asia

(A) Distribution of HIV-1 subtype B and CRF01_AE transmission clusters across seven countries in East and Southeast Asia. Size and frequency of transmission clusters were estimated based on the polymerase gene sequences (HXB2 position: 2252-3263 nt) from 506 and 226 MSM individuals infected with subtype B and CRF01_AE, respectively. In this study, transmission clusters are defined based on statistical supports generated at the internal nodes of the maximum likelihood and Bayesian's maximum clade credibility (MCC) tree reconstructions (bootstrap values of more than $90 \%$ and posterior probability of 1 , 
respectively). (B) Phylodynamic characteristics of HIV-1 subtype B and CRF01_AE transmission clusters. The Bayesian coalescent-based relaxed molecular clock analysis was performed as described elsewhere. ${ }^{15}$ The mean time of the most recent common ancestor (tMRCA), displayed in ascending order from the oldest to the youngest cluster, with the 95\% highest posterior distribution for each transmission network are shown. The year in which cART was first introduced is also indicated. 